\title{
A Novel Method for Profiling and Quantifying Short- and Medium- Chain Chlorinated Paraffins in Environmental Samples Using Comprehensive Two-Dimensional Gas Chromatography-Electron Capture Negative Ionization High-Resolution Time-of-Flight Mass Spectrometry
}

\author{
Dan Xia, ${ }^{\dagger}{ }^{\dagger}$ Lirong Gao, ${ }^{* \dagger}$ Minghui Zheng, ${ }^{\dagger}$ Qichang Tian, ${ }^{\dagger}$ Huiting Huang ${ }^{\dagger}$ and Lin Qiao ${ }^{\dagger}$ \\ ${ }^{\dagger}$ State Key Laboratory of Environmental Chemistry and Ecotoxicology, Research Center for Eco-Environmental Sciences, Chinese \\ Academy of Sciences, Beijing 100085, China \\ ${ }^{\ddagger}$ University of Chinese Academy of Sciences, Beijing 100085, China
}

\section{Supporting Information}

\begin{abstract}
Chlorinated paraffins (CPs) are complex technical mixtures containing thousands of isomers. Analyzing CPs in environmental matrices is extremely challenging. CPs have broad, unresolved profiles when analyzed by onedimensional gas chromatography (GC). Comprehensive twodimensional GC (GCXGC) can separate CPs with a high degree of orthogonality. A novel method for simultaneously profiling and quantifying short- and medium-chain CPs, using GCXGC coupled with electron capture negative ionization high-resolution time-of-flight mass spectrometry, was developed. The method allowed $48 \mathrm{CP}$ formula congener groups to be analyzed highly selectively in one injection through accurate mass measurements of the $[\mathrm{M}-\mathrm{Cl}]^{-}$ions in full scan mode.

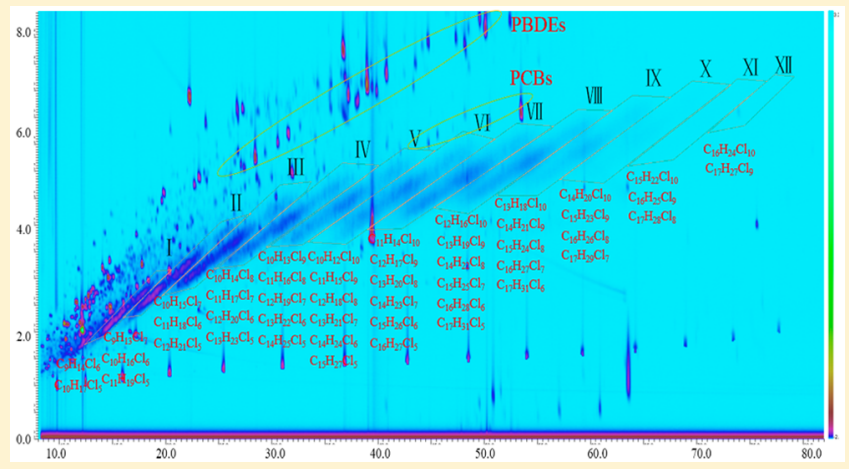

The correlation coefficients $\left(R^{2}\right)$ for the linear calibration curves for different chlorine contents were 0.982 for short-chain CPs and 0.945 for medium-chain CPs. The method was successfully used to determine CPs in sediment and fish samples. By using this method, with enhanced chromatographic separation and high mass resolution, interferences between $\mathrm{CP}$ congeners and other organohalogen compounds, such as toxaphene, are minimized. New compounds, with the formulas $\mathrm{C}_{9} \mathrm{H}_{14} \mathrm{Cl}_{6}$ and $\mathrm{C}_{9} \mathrm{H}_{13} \mathrm{Cl}_{7}$, were found in sediment and biological samples for the first time. The method was shown to be a powerful tool for the analysis of CPs in environmental samples.
\end{abstract}

\section{INTRODUCTION}

Chlorinated paraffins (CPs) are synthetic mixtures with chlorine contents usually ranging from $30 \%$ to $70 \%$ by mass. ${ }^{1}$ CPs are produced by chlorinating $n$-alkane feedstocks with molecular chlorine. ${ }^{1}$ On the basis of the carbon chain lengths, CPs can be divided into short-chain CPs (SCCPs, with carbon chain lengths of $\mathrm{C}_{10}-\mathrm{C}_{13}$ ), medium-chain CPs (MCCPs; $\mathrm{C}_{14}-$ $\mathrm{C}_{17}$ ), and long-chain $\mathrm{CPs}\left(\mathrm{C}_{>17}\right)$. Technical CP formulations are mainly used as additives in metal-working fluids and lubricants, as secondary plasticizers, and as flame retardants in polymeric materials. ${ }^{1,2}$ Attention should be paid to CPs because large amounts of CPs are produced (up to $10^{6} \mathrm{t}$ in China in 2009). ${ }^{3}$ Because of their toxicity, persistence, and potential to undergo long-range transport and to bioaccumulate, SCCPs have been listed as candidate persistent organic pollutants under the Stockholm Convention. ${ }^{4}$ MCCPs have properties similar to those of SCCPs; therefore more attention should be paid to the use of MCCPs. ${ }^{5,6}$ More comparable data on SCCP and MCCP concentrations in environmental media are needed urgently. However, methods for accurately analyzing CPs are not well developed because of the difficulties involved in separating and quantifying CPs. The complex compositions of $\mathrm{CP}$ mixtures and the different $\mathrm{CP}$ congener patterns found in different environmental matrices make analysis of CPs even more difficult than that of other chemicals. ${ }^{7}$ There are more than 10000 possible CP congeners. Some congeners have similar chromatographic retention characteristics. It is difficult to quantitatively analyze CP congener groups. ${ }^{8-10}$

Currently, the most widely used technique for CP analysis is one-dimensional (1D) gas chromatography (GC) coupled with electron capture negative ionization (ECNI) low-resolution quadrupole mass spectrometry (LRMS $)^{11-14}$ or sector field high-resolution mass spectrometry $(\mathrm{HRMS})^{7}$ in selected ion

Received: March 21, 2016

Revised: $\quad$ May 8, 2016

Accepted: May 16, 2016

Published: May 16, 2016 
monitoring mode. Some SCCP and MCCP congeners have the same GC retention times, and individual CP formula congener and total $\mathrm{CP}$ concentrations may be overestimated because of mass overlaps between different $\mathrm{CP}$ congeners. For example, the $[\mathrm{M}-\mathrm{Cl}]^{-}$ions of $\mathrm{C}_{11} \mathrm{H}_{17}{ }^{37} \mathrm{Cl}^{35} \mathrm{Cl}_{6}(\mathrm{~m} / z$ 395.9) and $\mathrm{C}_{16} \mathrm{H}_{29}{ }^{35} \mathrm{Cl}_{5}(m / z 396.1)$ will overlap when analyzed by ECNILRMS. ${ }^{14}$ Quantifying some MCCP congeners is not possible because of interference from SCCP congeners and vice versa. In addition, the physical and chemical properties of CPs are similar to those of other persistent organic pollutants, such as organochlorine pesticides (OCPs), polybrominated diphenyl ethers (PBDEs), polychlorinated biphenyls (PCBs), and toxaphenes, and these compounds may coelute with CPs and interfere with the quantitative analysis of CPs. ${ }^{5,14,15}$ Zeng et al. ${ }^{16}$ developed a quantification method to decrease interferences between CP homologues on the LRMS. In the method, each sample is injected four times to allow all of the selected $\mathrm{CP}$ congeners to be analyzed. These multiple injections may increase the errors involved in the analysis and increase the data-processing time. The results of international laboratory comparison studies using the methods mentioned above have shown that currently used CP analysis methods are far from satisfactory. ${ }^{17,18}$ Alternative methods using electron ionization tandem $\mathrm{MS}^{19}$ have been developed to allow the total amounts of CPs in samples to be determined, but it is not possible to identify congener and homologue group patterns using these methods. Bogdal et al. ${ }^{20}$ recently proposed a method in which CPs are directly analyzed by quadrupole time-of-flight (TOF) high-resolution MS combined with a mathematical patterndeconvolution algorithm. This method can be used to analyze $\mathrm{CPs}$ in environmental samples quickly, and is particularly sensitive for MCCPs and LCCPs.

Chromatographically separating the different CP congener groups will improve the accuracy with which CPs in environmental samples can be quantified. Effective chromatographic separation of $\mathrm{CP}$ congeners can potentially be achieved using two-dimensional GC $(\mathrm{GC} \times \mathrm{GC})$, in which two GC columns with different retention mechanisms are used. ${ }^{21}$ GC $\times$ GC has a higher peak capacity than any other chromatographic techniques; therefore, it can be used to separate complex organic mixtures and separate analytes from unresolved complex matrices. $^{22-24}$ Korytár et al. $^{25}$ used GC $\times$ GC-TOF-MS to separate and characterize CP mixtures. Different CP formula groups were not be completely separated using the limited column combinations and low-resolution instrument available to them. The quantification of CPs formula group in technical mixtures and environmental samples is not possible because of significant chromatographic overlaps. We have developed a method using GCXGC coupled to a microelectron capture detector for successfully separating complex mixtures of SCCPs with similar structures into groups. Separation was achieved in this method using a DM-1 column and a BPX-50 column. ${ }^{26}$ However, different CP formula groups could not be accurately identified and quantified because of the poor selectivity of the microelectron capture detector. With a high mass resolution and mass accuracy, HRTOF-MS could eliminate matrix interferences and significantly improve selectivity when analyzing CPs, giving more accurate analytical data. ${ }^{27}$ Moreover, rapid improvements in the software available for processing $\mathrm{GC} \times \mathrm{GC}$ data $^{28}$ have made it possible to use GCXGC-HRTOF-MS to solve the problems involved in quantifying CPs in environmental samples.
The objective of this study was to develop a new, sensitive, and effective GCXGC-ECNI-HRTOF-MS method for simultaneously profiling and quantifying SCCPs and MCCPs. The $\mathrm{CP}$ concentrations in sediment and fish samples were determined. Possible interferences caused by other CP congener groups and organochlorine compounds were evaluated. In addition, unknown new CPs were found in environmental samples.

\section{EXPERIMENTAL SECTION}

Chemicals and Standards. Three standard SCCP mixtures (with chlorine contents of $51.0 \%, 55.5 \%$, and 63.0\%) and three standard MCCP mixtures (with chlorine contents of $42.0 \%, 52.0 \%$, and $57.0 \%$ ), all at $100 \mathrm{ng} / \mu \mathrm{L}$ in cyclohexane, were obtained from Dr. Ehrenstorfer (Augsburg, Germany). Five CP mixtures with different chlorine contents were used to establish linear calibration curves [shown in detail in Table S1, Supporting Information (SI)]. ${ }^{13} \mathrm{C}_{10}$-Labeled transchlordane, purchased from Cambridge Isotope Laboratories (CIL), was added (to give a final concentration of $500 \mathrm{pg} / \mu \mathrm{L}$ ) to each calibration standard and sample to act as an internal standard. $\varepsilon$-Hexachlorocyclohexane, obtained from Dr. Ehrenstorfer, was used as a recovery standard. Interfering test mixtures include 27 OCP congeners (CIL), 39 PBDE congeners (AccuStandard Inc.), 72 PCB congeners (Wellington Laboratories, Guelph, Canada), and technical toxaphenes (Dr. Ehrenstorfer).

Sample Preparation. Five sediment samples from the middle reaches of the Yellow River, China, and five fish samples from Bohai Bay, China, were prepared and analyzed by GCXGC-ECNI-HRTOF-MS. Sample extraction and cleanup procedures that have previously been published, ${ }^{7,16}$ with some modifications, were used. The procedures are described in detail in the Supporting Information.

GC $\times$ GC-ECNI-HRTOF-MS Instrument. The GC $\times \mathrm{GC}-$ ECNI-HRTOF-MS analyses were conducted using an Agilent 7890A GC instrument (Agilent Technologies, Santa Clara, CA) fitted with a ZX2004 loop cryogenic modulator (Zoex Corp., Houston, TX) and connected to a HRTOF-MS instrument (Tofwerk, Thun, Switzerland), which was operated in ECNI mode with an electron energy of $125 \mathrm{eV}$. The data acquisition speed was $100 \mathrm{~Hz}$. The reagent gas was methane. The MS instrument was tuned to give a resolving power of 5000 and a mass accuracy of $5 \mathrm{ppm}$ or $0.002 \mathrm{u}$ (calculated using the full width at half-maximum), using perfluoroperhydrophenanthrene as the mass calibrant. The accurate masses of the main perfluoroperhydrophenanthrene ions produced by ECNI $(\mathrm{m} / z$ 261.9845, 280.9829, 311.9814, 361.9782, 404.9766, 423.9750, $461.9718,585.9654,604.9638$, and 623.9622) were used to perform a pulse mass calibration after each sample had been analyzed. The first-dimension column was a DB-5 ms column (30 $\mathrm{m}$ long, $0.25 \mathrm{~mm}$ i.d., with a $0.25-\mu \mathrm{m}$-thick $100 \%$ dimethyl polysiloxane film; Agilent Technologies), and the seconddimension column was a BPX-50 column ( $1 \mathrm{~m}$ long, $0.1 \mathrm{~mm}$ i.d., and with a $0.1-\mu$ m-thick $50 \%$ phenyl polysilphenylene siloxane film; SGE, Melbourne, Australia). The carrier gas was helium, and the constant flow rate was $1.0 \mathrm{~mL} / \mathrm{min}$. A $1-\mu \mathrm{L}$ portion of a sample was injected in splitless mode, and the injection port was at $280{ }^{\circ} \mathrm{C}$. The oven temperature program started at $140{ }^{\circ} \mathrm{C}$ (held for $1 \mathrm{~min}$ ), increased at $10{ }^{\circ} \mathrm{C} / \mathrm{min}$ to $200{ }^{\circ} \mathrm{C}$, and then increased at $1.5^{\circ} \mathrm{C} / \mathrm{min}$ to $310^{\circ} \mathrm{C}$ (held for 5 $\mathrm{min})$. The modulation period was $8 \mathrm{~s}$. The MS instrument transfer line temperature was $280{ }^{\circ} \mathrm{C}$, and the ion source 
temperature was $200{ }^{\circ} \mathrm{C}$. GC $\times$ GC data were viewed and processed using GC Image R2.5 software (GC Image, Lincoln, $\mathrm{NE)}$ with support for HR data.

\section{RESULTS AND DISCUSSION}

Optimization of the GC $\times$ GC and HRTOF-MS Parameters. The objective of the GCXGC optimization process was to achieve the best separation of multiple CP formula groups and good separation between the target $\mathrm{CP}$ compounds and matrix components. Three sets of column combinations [DM-1 (a nonpolar column with $100 \%$ methylpolysiloxane stationary phase) and BPX-50, DM-1 and BPX-70, and DM-1 and DBWAX] were tested to maximize the chromatographic separation of SCCPs. ${ }^{26}$ The results showed that the DM-1 and BPX-50 combination separated the SCCPs well. A DB-5 ms column is also a nonpolar column that is widely used in 1D GC analysis of CPs. A DB-5 ms column will give similar results to a DM-1 column but will be more stable. DB-5 ms and BPX-50 columns were therefore subsequently used to analyze SCCPs and MCCPs. The first nonpolar DB-5 ms column separated the congeners by their boiling points and the second midpolar BPX-50 column mainly by polarity. The retention time on the first column increased as the length of the $\mathrm{CP}$ carbon chain increased, and the retention time on the second column increased as the polarity of the CP congener group increased. The selected column combination gave an orthogonal separation between the different CP formula congener groups and could separate the complex mixtures of $\mathrm{CP}$ congener groups in technical mixtures and environmental extracts well.

The length of the second column and the modulation period were optimized to give good separations of SCCPs and MCCPs in the second dimension. As can be seen in Figure 1A, a long second column ( $2 \mathrm{~m}$ long, $0.1 \mathrm{~mm}$ i.d., $0.1 \mu \mathrm{m}$ film thickness) and short modulation period ( $8 \mathrm{~s}$ ) caused "wrap-around" (some CP congeners, especially MCCP congeners, coeluted with the tuning standard at the beginning of the next modulation period). Some formula congeners, such as $\mathrm{C}_{14} \mathrm{H}_{21} \mathrm{Cl}_{9}, \mathrm{C}_{14} \mathrm{H}_{20} \mathrm{Cl}_{10}$, and $\mathrm{C}_{15} \mathrm{H}_{24} \mathrm{Cl}_{8}$, also coeluted with the column bleed peak band (Figure $1 \mathrm{~A})$. Moreover, in environmental samples, wrap-around may cause longer chain and higher chlorinated CPs in the next modulation period to coelute with coextracted matrix components. No wrap-around was observed when a longer modulation period (10 s) was used (Figure 1B), but some SCCP and MCCP congeners overlapped because the separation efficiency of the first column decreased when a longer modulation period was used. With a short second column ( $1 \mathrm{~m}$ long, $0.1 \mathrm{~mm}$ i.d., $0.1 \mu \mathrm{m}$ film thickness) and a short modulation period ( $8 \mathrm{~s})$, different SCCP and MCCP congener groups were separated well, as shown in Figure 1C. For the technical SCCP and MCCP mixture, more than 2300 peaks could be distinguished in the GCXGC chromatogram using the optimized parameters. The CPs were separated into formula congener groups, each group having $\mathrm{CP}$ formula congeners with different carbon chain lengths and different chlorine atoms.

The sensitivities achieved using different ion source temperatures when analyzing CPs by ECNI MS have been assessed in previous studies, ${ }^{, 29}$ and the relative abundances of the $[\mathrm{M}-$ $\mathrm{Cl}]^{-}$ions were found to decrease as the source temperature increased. An ion source temperature of $200{ }^{\circ} \mathrm{C}$ in this study was selected as a compromise between achieving the best possible sensitivity and minimizing contamination by repeatedly analyzing samples. The methane flow rate and TOF-MS
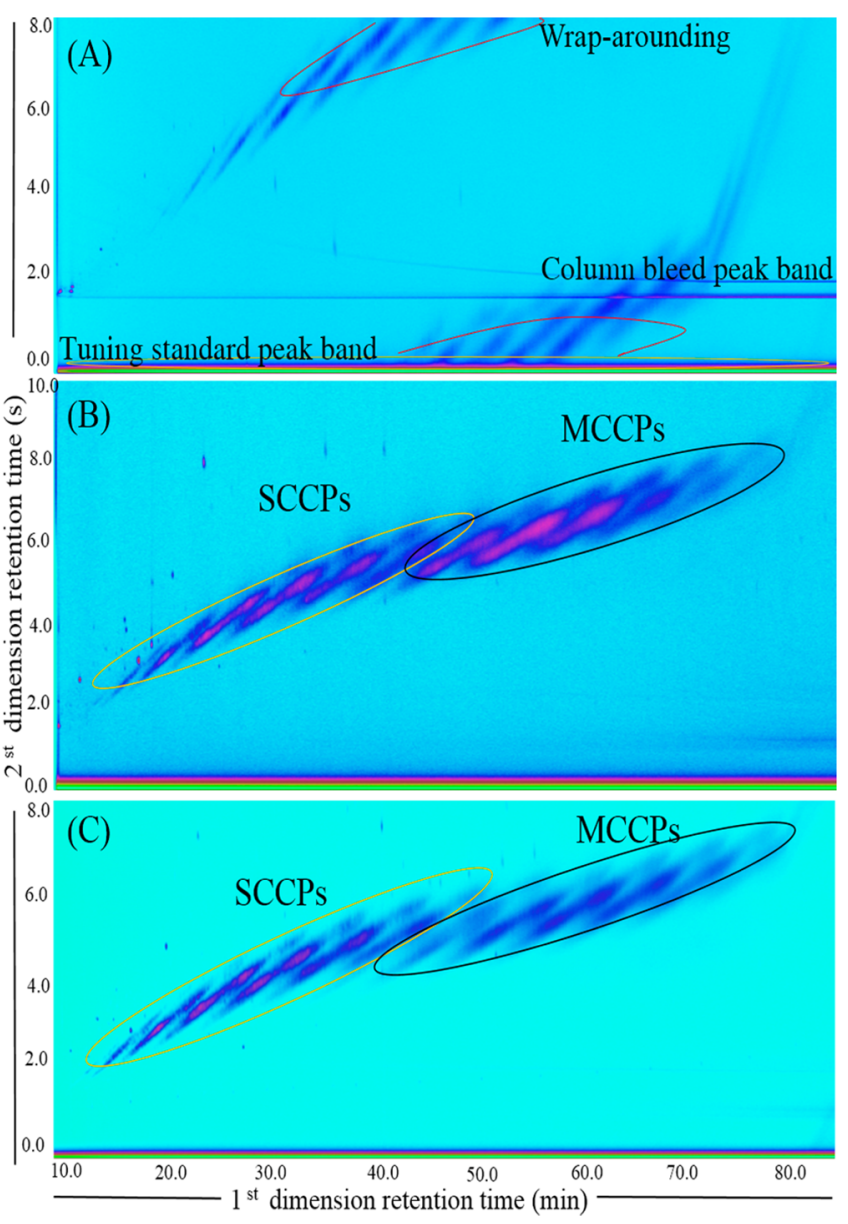

Figure 1. GC $\times$ GC-ECNI-HRTOF-MS total ion chromatogram (TIC) of a mixture of a technical SCCP mixture (containing $51.5 \%$ $\mathrm{Cl}, 10 \mathrm{ng} / \mu \mathrm{L}$ ) and a technical MCCP mixture (containing $52 \% \mathrm{Cl}, 10$ $\mathrm{ng} / \mu \mathrm{L}$ ), acquired using second columns of different lengths and using different modulation periods: (A) using a second column $2 \mathrm{~m}$ long and a modulation period of $8 \mathrm{~s}$, (B) using a second column $2 \mathrm{~m}$ long and a modulation period of $10 \mathrm{~s}$, and (C) using a second column $1 \mathrm{~m}$ long and a modulation period of $8 \mathrm{~s}$.

parameters also strongly influenced the sensitivity and were optimized for the mass range of interest $(\mathrm{m} / z$ 50-650). A GCXGC data acquisition rate of $100 \mathrm{~Hz}$ was selected to allow reproducible signals (with relative standard deviations of $2 \%-$ $10 \%$ for areas and $0.2 \%-0.3 \%$ for retention times, with peak widths in the second dimension of $300 \mathrm{~ms}$ ) to be obtained. It should be noted that, when GCXGC-ECNI-HRTOF-MS analysis are performed, a computer with a large storage capacity is required because each data file will be 5-10 Gb. Also, the large amounts of data produced when CPs are analyzed need to be manipulated with high expertise in data handling and processing.

Profiling the Elution Patterns of CP Formula Congener Groups. The GCXGC TIC chromatogram of a mixture of technical $51.5 \% \mathrm{Cl}$ SCCP and technical $52 \% \mathrm{Cl}$ MCCP was acquired and is shown in Figure 2A. The CP formula congener groups were markedly better separated by GC $\times$ GC than by $1 \mathrm{D}$ GC, in which all CP congeners eluted as a broad and unresolved peak (Figure S1, SI). Different CP formula congener groups were effectively separated by $\mathrm{GC} \times \mathrm{GC}$ (Figure S2, SI), and the CP elution pattern is shown in Figure 2A. The SCCPs eluted quickly from the first column, and 


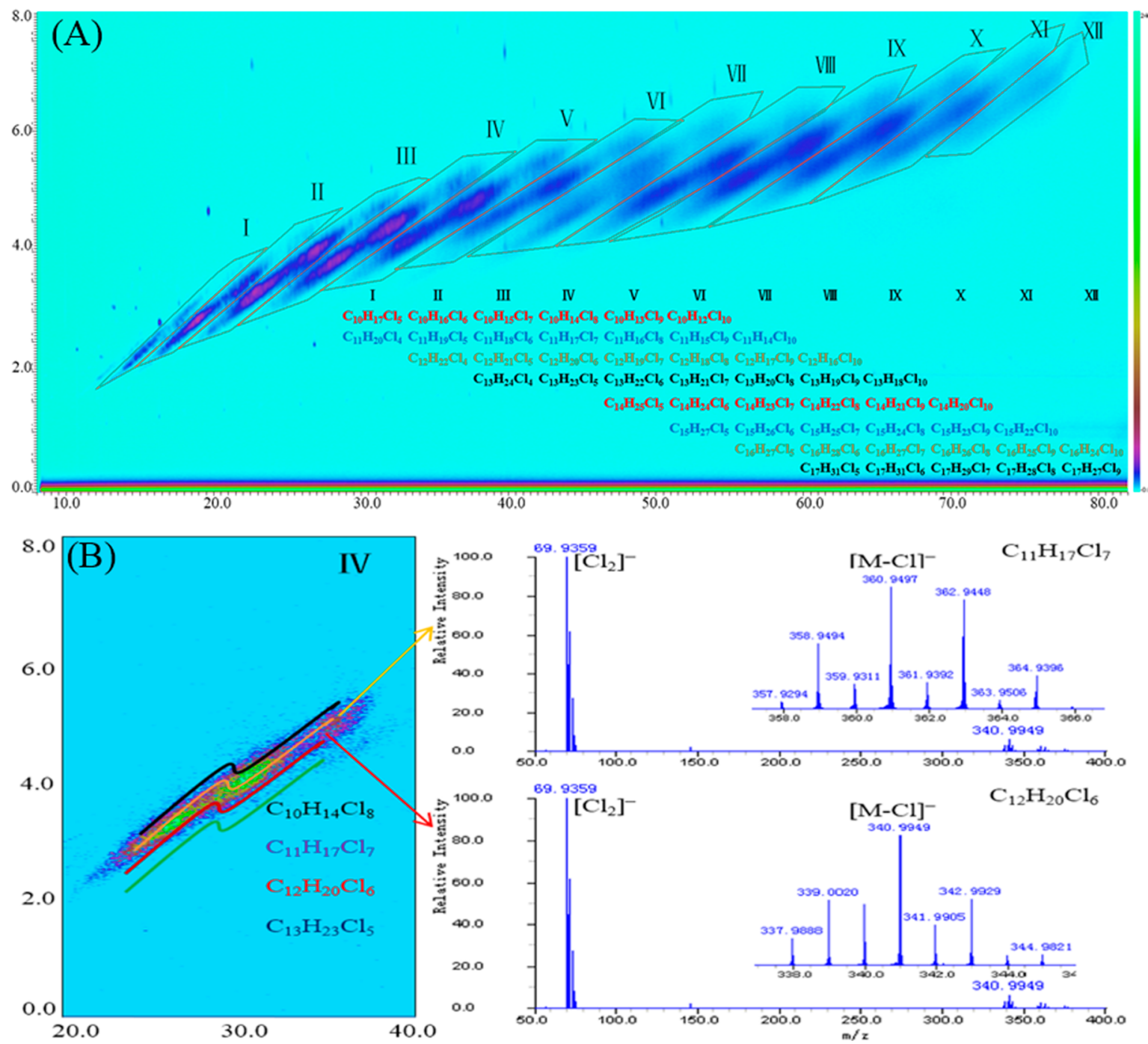

Figure 2. (A) GC $\times$ GC-ECNI-HRTOF-MS TIC chromatograms and GC $\times$ GC elution pattern for a mixture of a technical $51.5 \% \mathrm{Cl}$ SCCPs mixture and a technical 52\% $\mathrm{Cl}$ MCCPs mixture (lower right: molecular formulas of the $\mathrm{CP}$ congeners in each group). (B) Extracted ion chromatograms (left: group IV containing $\mathrm{CP}$ formula congeners of $\mathrm{C}_{10} \mathrm{H}_{14} \mathrm{Cl}_{8}, \mathrm{C}_{11} \mathrm{H}_{17} \mathrm{Cl}_{7}, \mathrm{C}_{12} \mathrm{H}_{20} \mathrm{Cl}_{6}$, and $\mathrm{C}_{13} \mathrm{H}_{23} \mathrm{Cl}_{5}$ ) and accurate mass spectra (right) of selected peaks for CPs analyzed using the GCXGC-ECNI-HRTOF-MS.

prominent, regularly spaced peaks were found. The MCCPs eluted later than the SCCPs. The GC $\times$ GC chromatogram contained ordered structures on diagonal lines, and each structure was formed by a group of CPs with the same total number of carbon atoms and chlorine atoms. ${ }^{25,26}$ The separation pattern was confirmed by extracting ion chromatograms and accurate mass spectra. As shown in Figure 2B, the extracted ion chromatograms of group IV were obtained. The $\mathrm{CP}$ formula congeners $\mathrm{C}_{10} \mathrm{H}_{14} \mathrm{Cl}_{8}, \mathrm{C}_{11} \mathrm{H}_{17} \mathrm{Cl}_{7}, \mathrm{C}_{12} \mathrm{H}_{20} \mathrm{Cl}_{6}$, and $\mathrm{C}_{13} \mathrm{H}_{23} \mathrm{Cl}_{5}$ were all in the same group, with an accurate mass $\mathrm{m}$ / $z$ corresponding to $[\mathrm{M}-\mathrm{Cl}]^{-}$. The boiling point selectivity of the first column and polarity selectivity of the second column allowed separation of CP congener groups with different carbon chain lengths and different chlorine atoms. The group separation patterns for all of the SCCP and MCCP congeners can clearly be seen in the GCXGC chromatogram shown in Figure $2 \mathrm{~A}$, in which the molecular formulas of the congeners in each group are shown on the lower right. The GC $\times$ GC contour plot contained 12 groups, labeled I-XII on the chromatogram, and a polygon is drawn around each group of congeners with the same total number of carbon and chlorine atoms. The groups that are marked were convenient for identifying the selected CP formula congeners, but other CP formula congener groups in different matrices could also be identified from the accurate masses found. With GC $\times$ GC mass chromatogram of an $\mathrm{m} / z 0.02$ wide window around the accurate mass for a selected CP formula congener, SCCP and MCCP congener profiles of the technical $\mathrm{CP}$ mixture were acquired. The $\mathrm{CP}$ congener groups were well-separated using the optimized conditions described above, and the $\mathrm{CP}$ formula congener groups could then be quantified using the group pattern analysis strategy described here.

We focused on the analysis of SCCP and MCCP congeners with 10-17 carbon atoms and 5-10 chlorine atoms. A total of $48 \mathrm{CP}$ formula congeners with the same total number of carbon and chlorine atoms (e.g., $\mathrm{C}_{10} \mathrm{H}_{17} \mathrm{Cl}_{5}$ ) were analyzed in one injection. Several mono-, di-, tri-, and tetrachlorinated individual CP congeners were analyzed by GCXGC-ECNITOF-MS and were found to give $[\mathrm{M}-\mathrm{Cl}]^{-}$peaks with rather low responses. Similar results have previously been found using ECNI-quadrupole MS. ${ }^{7} \mathrm{CP}$ congeners with fewer than five chlorine atoms might be present in environmental samples, but 

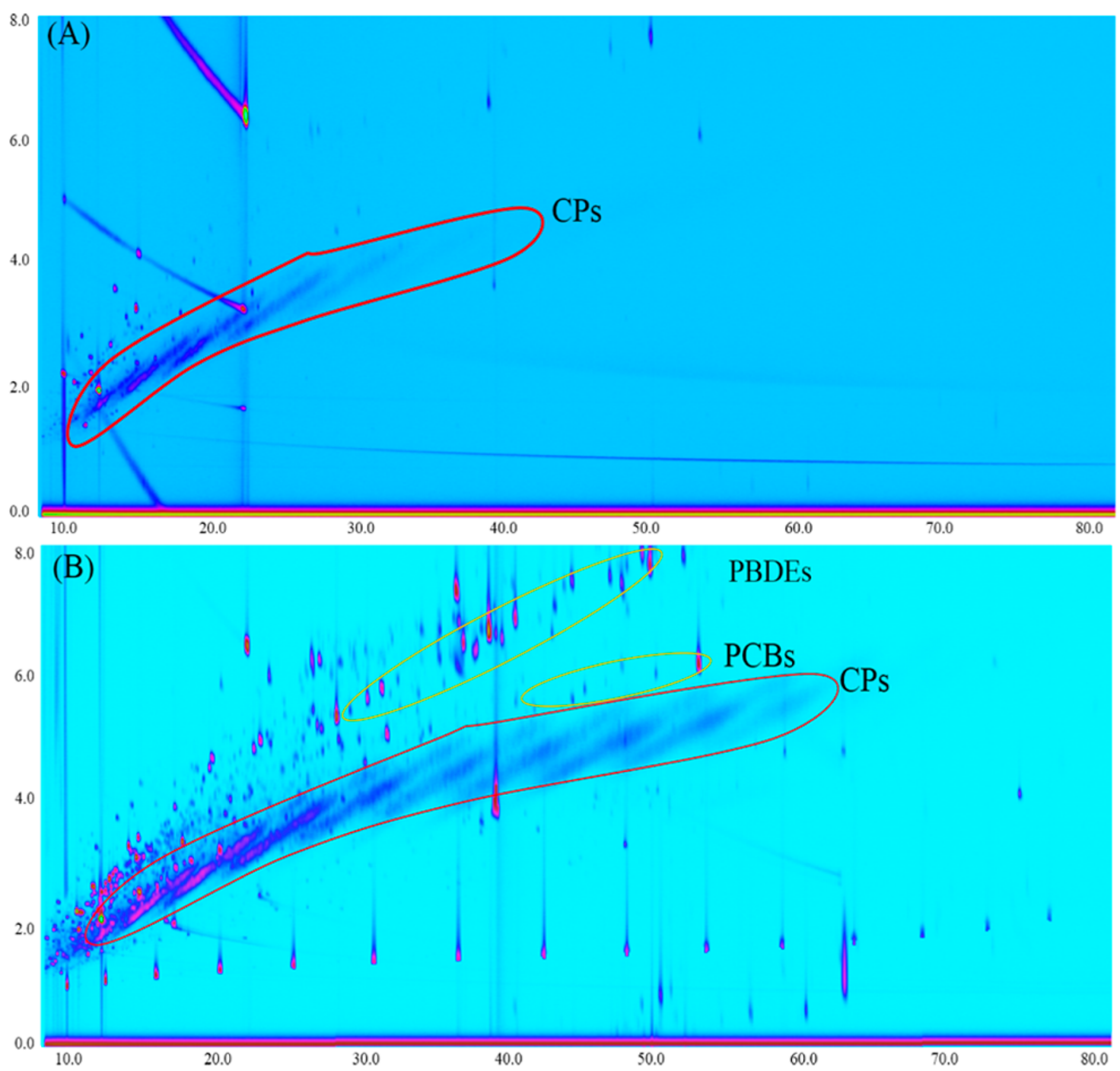

Figure 3. GC $\times$ GC-ECNI-HRTOF-MS TIC chromatograms of (A) a sediment sample and (B) a fish sample.

they cannot usually be analyzed by ECNI-TOF-MS because of their low responses. We did not attempt to quantify these compounds. In general, ECNI mainly causes CPs to form $[\mathrm{M}-$ $\mathrm{Cl}]^{-},[\mathrm{M}-\mathrm{HCl}]^{-}$, and $[\mathrm{M}+\mathrm{Cl}]^{-}$ions. The $[\mathrm{M}+\mathrm{Cl}]^{-}$ion was the base ion in the 1,2,9,10-tetrachlorodecane mass spectrum, but the $[\mathrm{M}+\mathrm{Cl}]^{-}$intensity decreased to about $60 \%$ in the 1,2,5,6,9-pentachlorodecane mass spectrum and about $10 \%$ in the $1,2,4,5,9,10$-hexachlorodecane mass spectrum (Figure S3, SI). The $[\mathrm{M}-\mathrm{Cl}]^{-}$ion was the most abundant in the mass spectra of CPs with formulas between $\mathrm{C}_{10} \mathrm{H}_{17} \mathrm{Cl}_{5}$ and $\mathrm{C}_{17} \mathrm{H}_{26} \mathrm{Cl}_{10}$ that have been found in large amounts in environmental samples. The accurate masses and abundances (as percentages of the total ion formula abundances) of the [M $-\mathrm{Cl}]^{-}$ions of selected analyzed CPs congener groups are given in Table S2 (SI).

The results shown in Figure 2 indicate that the CP congener group profiles and elution patterns could be accurately identified using the GC $\times$ GC-HRTOF-MS method. Different $\mathrm{CP}$ congener groups were separated well, and this will improve the accuracy of analyzing congener group patterns in environmental samples from different sources. The method may therefore improve our ability to identify the sources of CPs in the environment.
Development of the Quantification Procedure. The response factor of a CP congener when ECNI-MS is used is strongly dependent on the number of chlorine atoms the $\mathrm{CP}$ contains. Quantification results may be incorrect if the CP congener patterns in technical mixture standards are different from the patterns in the environmental samples being analyzed. ${ }^{12,30}$ Zencak et al. ${ }^{30}$ showed that ECNI-MS analysis results could deviate by more than $100 \%$ when the $\mathrm{CP}$ congener patterns in technical mixture standards are different from that in environmental samples. Reth et al. ${ }^{12}$ described a quantification method that compensates for different total response factors of SCCPs caused by the samples and standards having different chlorine contents. A linear correlation between the total response factors for CP standard mixtures and the chlorine contents of the mixtures can be obtained when a series of CP standards with different chlorine contents is analyzed.

The method we used to quantify SCCPs and MCCPs was based on the principles developed by Reth et al. ${ }^{12}$ It has been shown in previous studies that $[\mathrm{M}-\mathrm{Cl}]^{-}$is suitable for quantifying SCCPs in environmental samples. ${ }^{7,11,31} \mathrm{We}$ quantified MCCPs in a similar way to SCCPs. For each CP formula congener with 5-10 chlorine atoms, the highest most abundant $[\mathrm{M}-\mathrm{Cl}]^{-}$ion was used as a quantification ion and the next most abundant ion was used as a qualification ion. The 

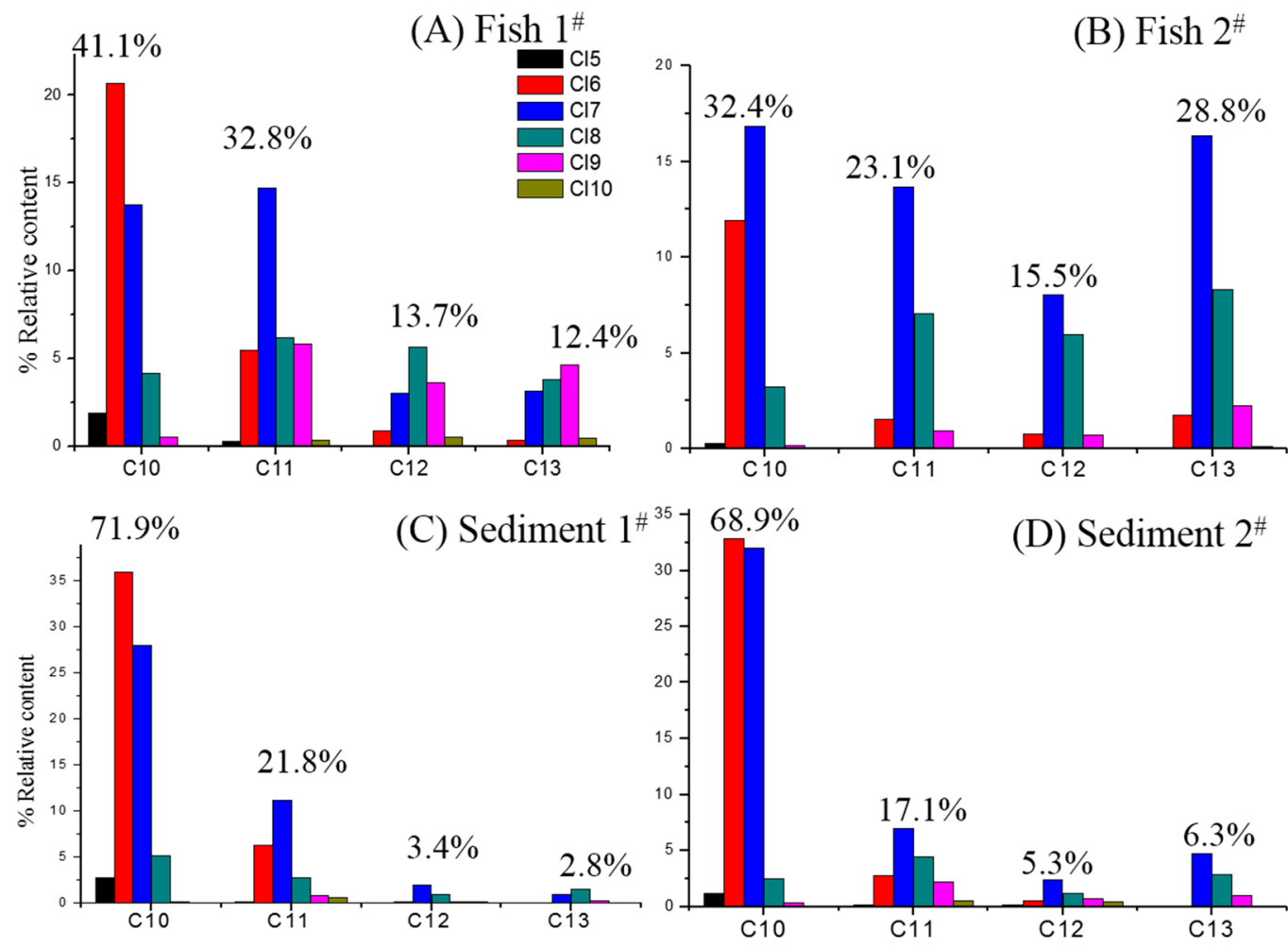

Figure 4. SCCP congener group abundance profiles for (A) fish 1, (B) fish 2, (C) sediment 1, and (D) sediment 2 samples, determined by GCXGC-ECNI-HRTOF-MS. The percentages above the chart are the total relative abundance for each carbon chain group.

selected quantification and qualification ions are shown in Table S2 (SI). All 48 SCCP and MCCP formula congeners were able to be simultaneously analyzed in full scan mode in one injection using the method. The formula congeners were divided into 12 groups (labeled I-XII in Figure 2A), each containing congeners with the same total number of carbon and chlorine atoms (e.g., $\mathrm{C}_{10} \mathrm{H}_{15} \mathrm{Cl}_{7}, \mathrm{C}_{11} \mathrm{H}_{18} \mathrm{Cl}_{6}$, and $\mathrm{C}_{12} \mathrm{H}_{21} \mathrm{Cl}_{5}$ were quantified in the same group).

The quantification procedure that was performed in combination with the group analysis strategy involved using eqs $1-5$ (shown in the Supporting Information). The first step was to obtain the $[\mathrm{M}-\mathrm{Cl}]^{-}$response for each formula congener (e.g., $\mathrm{C}_{10} \mathrm{H}_{15} \mathrm{Cl}_{7}$ ). The relative total response was then calculated by dividing the congener group response by the internal standard response. Standard CP solutions with different chlorine contents were analyzed, and the chlorine content of the CPs in a sample was calculated using eq 3 (SI). Calibration curves were established between the total response factor for a CP mixture and the calculated chlorine content for five CPs with different chlorine contents $(53.50 \%-59.25 \%$ for SCCPs and $44.00 \%-57.00 \%$ for MCCPs), as shown in eq 4 (SI). The total response factor for the CPs in a sample was then determined from the chlorine content using the calibration curves. The total amounts of SCCPs and MCCPs in the sample were calculated from the ratio between the total relative response of the sample and the corrected total response factor, using eq 5 (SI).

Linearity of the Response, Detection Limits, and Accuracy of the Method. A calibration curve was obtained between the total response factors and chlorine contents of the standards. The SCCPs had chlorine contents of 53.50-59.25\%, and the MCCPs had chlorine contents of $44.00-57.00 \%$. The correlation coefficients $\left(R^{2}\right)$ for the SCCPs and MCCPs were
0.982 and 0.945, respectively (shown in Figure S4, parts A and $\mathrm{B}$, respectively). The $R^{2}$ obtained using HRTOF-MS agreed well with results that have been obtained using quadrupole MS. ${ }^{12}$ The relative standard deviations of the slopes and intercepts (determined by performing three replicate injections) were less than $20 \%$. The analytical detection limit (the amount of analyte giving a signal-to-noise ratio of 3) was $20 \mathrm{pg}$ for total SCCPs $(55.5 \%$ chlorine content, detection of the most abundant congener group $\mathrm{C}_{11} \mathrm{H}_{18} \mathrm{Cl}_{6}$ ) and $100 \mathrm{pg}$ for MCCPs (52\% chlorine content, detection of the most abundant congener group $\mathrm{C}_{14} \mathrm{H}_{23} \mathrm{Cl}_{7}$ ). Procedural blanks were analyzed, and the blanks contained, on average, $5.8 \mathrm{ng}$ of SCCPs and 4.8 ng of MCCPs. The method detection limit was defined as the average amount of CPs in the blanks plus 3 times the standard deviation. The estimated method detection limits were 3.7 and $2.1 \mathrm{ng} / \mathrm{g}$ for the SCCPs and MCCPs, respectively, in sediment, and 9.4 and $7.0 \mathrm{ng} / \mathrm{g}$ for the SCCPs and MCCPs, respectively, in fish. The method detection limits were lower than or comparable to those achieved using HRGC-ECNI-HRMS (23 $\mathrm{ng} / \mathrm{g}){ }^{7}$ the method is therefore suitable for determining CPs in environmental samples. The average recoveries of $1 \mu \mathrm{g}$ of the $55.5 \% \mathrm{Cl}$ SCCP standard spiked into three sediment and fish samples were $92.5 \%$, and the average recoveries of $1 \mu \mathrm{g}$ of the $52 \% \mathrm{Cl}$ MCCP standard spiked into these samples were $90.3 \%$. The recoveries of ${ }^{13} \mathrm{C}_{10}$-labeled trans-chlordane spiked into five sediment and five fish samples were 65.0-92.0\%.

Analysis of CPs in Sediment and Fish Samples. The method was used to analyze SCCPs and MCCPs in five sediment and five fish samples to demonstrate that the method was suitable for analyzing CPs in different environmental matrices. The sediment samples were collected from the middle reaches of the Yellow River (the second largest river in China), which runs though many cities and finally enters Bohai Bay. 
The fish samples were collected in Bohai Bay. The CP congeners in one sediment and one fish sample are shown in the GC $\times$ GC TIC chromatograms in Figure 3A,B. The CP concentrations and congener distributions were different in different sediment and fish samples.

The SCCP and MCCP congener concentrations in the samples are summarized in Table S3 (SI). The total SCCP concentrations in the sediment samples were 66-490.8 ng/g dry weight $(\mathrm{dw})$, and the total MCCP concentrations were 20.5-93.7 ng/g dw. In general, the SCCP concentrations in the sediment samples were similar to concentrations found in sediment from the Liaohe River Basin $(39.8-480.3 \mathrm{ng} / \mathrm{g} \mathrm{dw})^{32}$ but lower than those found in sediment from the Pearl River Delta $(320-6600 \mathrm{ng} / \mathrm{g} \mathrm{dw}){ }^{33}$ The SCCP concentrations in the five fish samples were $373.6-3997 \mathrm{ng} / \mathrm{g} \mathrm{dw}$, and the MCCP concentrations were $42.1-5307 \mathrm{ng} / \mathrm{g} \mathrm{dw}$. Different SCCP concentrations were found in different types of fish, and the concentrations were comparable to that previously found in mollusks $(64.9-5510 \mathrm{ng} / \mathrm{g}) .^{34}$ The high SCCP and MCCP concentrations in some of the samples may be related to $\mathrm{CP}$ emissions from sources nearby.

The SCCP and MCCP congener group distributions in the samples were investigated. The SCCP congener group patterns in two sediment and two fish samples are shown in Figure 4. Similar SCCP congener group patterns were found in the sediment and fish samples. The $\mathrm{C}_{10} \mathrm{CPs}$ with six or seven chlorine atoms were the predominant congener group. The $\mathrm{C}_{10}$ and $\mathrm{Cl}_{6} \mathrm{CP}$ congeners are more volatile and water-soluble, so they may more easily migrate to remote regions through longrange atmospheric or waterborne transport than longer chain and higher chlorinated CPs. The SCCP patterns were similar to those found in the atmosphere in northern China ${ }^{35}$ and in marine sediment from the East China Sea. ${ }^{36}$ However, the patterns were different from those found in sediment from highly industrialized areas. ${ }^{33}$ Different CP congener patterns in different matrices indicated that the different matrices may have been contaminated through different CPs sources and/or that the CPs may have been subjected to different environmental processes. The MCCP congener group patterns in two sediment and two fish samples are shown in Figure S5 (SI). The $\mathrm{C}_{14}$ CPs were the predominant MCCPs in all of the sediment and fish samples, which is similar to the MCCPs patterns found in technical CP formulations.

Exclusion of Potential Interferences. The two main types of interference that occur in CP analysis are interferences between different $\mathrm{CP}$ congeners and interferences from other environmental contaminants. As is mentioned above, CP congeners with the same total number of carbon and chlorine atoms were in the same group in our GC $\times \mathrm{GC}$ chromatograms. We determined whether CPs with different formulas in the same group would interfere with each other. For example, $\mathrm{C}_{11} \mathrm{H}_{17} \mathrm{Cl}_{7}$ and $\mathrm{C}_{13} \mathrm{H}_{23} \mathrm{Cl}_{5}$ will be in the same group, and the $\mathrm{C}_{11} \mathrm{H}_{17} \mathrm{Cl}_{7}[\mathrm{M}-\mathrm{Cl}]^{-}$ion will interfere with the $\mathrm{C}_{13} \mathrm{H}_{23} \mathrm{Cl}_{5}$ $[\mathrm{M}]^{-}$ion when LRTOF-MS is used because LRTOF-MS only has a resolution of 1000 and both ions have an $\mathrm{m} / z$ ratio of 361. The HRTOF-MS instrument we used provided accurate mass measurements with a very narrow mass ratio window $(\mathrm{m} /$ $z 0.02$ ), so the $\mathrm{C}_{11} \mathrm{H}_{17} \mathrm{Cl}_{7}[\mathrm{M}-\mathrm{Cl}]^{-}$and $\mathrm{C}_{13} \mathrm{H}_{23} \mathrm{Cl}_{5}[\mathrm{M}]^{-}$ions were separated well.

We also determined whether some organic chemicals with similar physical and chemical properties (OCPs, PBDEs, PCBs, and toxaphenes) would interfere with the quantification of CPs. A mixture of 27 OCP congeners, 39 PBDE congeners, $72 \mathrm{PCB}$ congeners, technical toxaphenes, SCCPs $(51.5 \% \mathrm{Cl})$, and MCCPs $(57 \% \mathrm{Cl})$ was analyzed. The resolution offered by GC $\times$ GC allowed the CPs to be separated clearly from the other classes of compounds in full scan mode, as shown in Figure S6 (SI). SCCPs and MCCPs could be quantified without the other compounds interfering. Additionally, as many compounds may be coeluted with $\mathrm{CP}$ in the same sample extract fraction and would interfere with the quantification of the CPs, we determined whether other potentially interfering compounds could be completely separated from the CPs in analyzed sediment and fish samples. A GC $\times$ GC chromatogram of the CPs in a fish sample is shown in Figure $3 \mathrm{~B}$. The second dimension of separation achieved by GCXGC excluded these potentially interfering compounds. Chlordane and toxaphenes were not detected in the fish samples. A range of PBDEs and PCBs congeners were detected in the fish samples. PBDEs were retained longer than the CPs and PCBs on the second GC column. We concluded that the increased separation power offered by GCXGC and the accurate mass resolution offered by HRTOF-MS allowed accurate results to be obtained for different $\mathrm{CP}$ formula congeners.

Discovery of New CPs with Carbon Chain Lengths of Less than 10 in Environmental Samples. SCCPs are defined as CPs with carbon chains of $\mathrm{C}_{10}-\mathrm{C}_{13}$. The toxicities of CPs may increase as the carbon chain becomes shorter and more chlorine atoms are present. ${ }^{1}$ No previous reports of CPs with carbon chains shorter than 10 being found in sediment or biological samples are available. We found a structured group of compounds that eluted before the SCCPs in the first dimensions of the GC $\times \mathrm{GC}$ chromatograms. The orthogonal $\mathrm{CP}$ distribution patterns in the GCXGC chromatograms (ordered structures in the formula congener groups and the roof-tile effect for congeners) allowed new CPs to be identified. GC $\times$ GC extract chromatograms were acquired using MS with a 0.02-Da-wide window, and formula calculations were performed on the accurate mass molecular ions of $[\mathrm{M}-\mathrm{Cl}]^{-}$to attempt to identify the unknown compounds. The mass spectra of the unknown compounds had dominant $\mathrm{m} / z$ ratios of 332.9124 and 298.9514, which were found to be the $[\mathrm{M}-\mathrm{Cl}]^{-}$ peaks of $\mathrm{C}_{9} \mathrm{H}_{13} \mathrm{Cl}_{5}{ }^{37} \mathrm{Cl}^{-}$and $\mathrm{C}_{9} \mathrm{H}_{14} \mathrm{Cl}_{4}{ }^{37} \mathrm{Cl}^{-}$, respectively. The measured characteristic accurate mass spectra of the two compounds are shown in Figure S7 (SI), and the accurate $m / z$ values are shown in Table 1 , along with the calculated

Table 1. Accurate Masses Measurement of the Predominant Ions of the Newly Identified Compounds

\begin{tabular}{ccccc} 
& \multicolumn{4}{c}{ most abundant ion } \\
\cline { 2 - 5 } formula & {$[\mathrm{M}-\mathrm{Cl}]^{-}$} & $\begin{array}{c}\text { theoretical } \\
\mathrm{m} / z\end{array}$ & $\begin{array}{c}\text { measured } \\
\mathrm{m} / z\end{array}$ & $\begin{array}{c}\text { mass error } \\
(\mathrm{ppm})\end{array}$ \\
$\mathrm{C}_{9} \mathrm{H}_{13} \mathrm{Cl}_{7}$ & $\mathrm{C}_{9} \mathrm{H}_{13} \mathrm{Cl}_{5}{ }^{37} \mathrm{Cl}^{-}$ & 332.9119 & 332.9124 & 1.5 \\
$\mathrm{C}_{9} \mathrm{H}_{14} \mathrm{Cl}_{6}$ & $\mathrm{C}_{9} \mathrm{H}_{11} \mathrm{Cl}_{4}{ }^{37} \mathrm{Cl}^{-}$ & 298.9508 & 298.9514 & 2.0 \\
\hline
\end{tabular}

elemental compositions and the confirmed molecular formulas of the compounds. The mass accuracies of the measured ions were good, at less than a $2 \mathrm{ppm}$ mass error in each case, supporting our tentative identification of the two CPs as $\mathrm{C}_{9} \mathrm{H}_{13} \mathrm{Cl}_{7}$ and $\mathrm{C}_{9} \mathrm{H}_{14} \mathrm{Cl}_{6}$.

This is the first time new CPs with the formulas $\mathrm{C}_{9} \mathrm{H}_{13} \mathrm{Cl}_{7}$ and $\mathrm{C}_{9} \mathrm{H}_{11} \mathrm{Cl}_{6}$ have been found in sediment and biological samples. Finding these $\mathrm{C}_{9} \mathrm{CPs}$ in both matrices suggests that they could be present in industrial CP products and be released to the environment. CPs are produced through the free radical 
chlorination of paraffin feedstocks, meaning that a broad range of CPs are produced. Also, it is possible that the new CPs were produced through biotransformation or metabolism. On the whole, this discovery of new CPs will improve our understanding of CPs. Further investigations into their occurrences and behaviors in the environment are required, and the risks posed by these new CPs need to be assessed.

\section{ASSOCIATED CONTENT}

\section{S Supporting Information}

The Supporting Information is available free of charge on the ACS Publications website at DOI: 10.1021/acs.est.6b01404.

Sample preparation procedures, the quantification equations, Figures S1-S7, and Tables S1-S3 (PDF)

\section{AUTHOR INFORMATION}

\section{Corresponding Author}

*Tel: +861062849356; fax: +861062923563; e-mail: gaolr@ rcees.ac.cn.

\section{Author Contributions}

All authors contributed to the writing of the manuscript. All authors have approved the final version of the manuscript.

\section{Notes}

The authors declare no competing financial interest.

\section{ACKNOWLEDGMENTS}

This research was funded by the National Natural Science Foundation of China (Nos. 21377140, 213111064, and 21321004) and the Strategic Priority Research Program of the Chinese Academy of Sciences (XDB14020102). We appreciate the kind support and technical help related to the GCXGC system provided by Dr. Zhanpin Wu (Zoex Corp.), Bin Wang and Xiaolong Jiang (Polytech Instrument Corp.).

\section{REFERENCES}

(1) De Boer, J.; El-Sayed Ali, T.; Fiedler, H.; Legler, J.; Muir, D. C.; Nikiforov, V. A.; Tomy, G. T.; Tsunemi, K. Chlorinated Paraffins. In The Handbook of Environmental Chemistry, Chlorinated Paraffins; De Boer, J., Ed.; Springer-Verlag Berlin: Berlin, 2010; Vol. 10.

(2) Nicholls, C. R; Allchin, C. R.; Law, R. J. Levels of short and medium chain length polychlorinated $\mathrm{n}$-alkanes in environmental samples from selected industrial areas in England and Wales. Environ. Pollut. 2001, 114 (3), 415-430.

(3) Tong, X. C.; Hu, J. X.; Liu, J. G.; Wan, D. Environmental exposure analysis and screening risk assessment of short chain chlorinated paraffias in China. PINYIN: Huan Jing Ke Xue Yu Ji Shu (Environ. Sci. Technol.) 2009, 32, 438-441 (in Chinese).

(4) UNEP. Proposal on Next Steps for Short-Chained Chlorinated Paraffins UNEP/POPS/POPRC.8/16/Annex IV; Stockholm Convention on Persistent Organic Pollutants, Geneva, 2012.

(5) Santos, F. J.; Parera, J.; Galceran, M. T. Analysis of polychlorinated $\mathrm{n}$-alkanes in environmental samples. Anal. Bioanal. Chem. 2006, 386 (4), 837-857.

(6) Houde, M.; Muir, D. C. G.; Tomy, G. T.; Whittle, D. M.; Teixeira, C.; Moore, S. Bioaccumulation and trophic magnification of short- and medium-chain chlorinated paraffins in food webs from Lake Ontario and Lake Michigan. Environ. Sci. Technol. 2008, 42 (10), 3893-3899.

(7) Tomy, G. T.; Stern, G. A.; Muir, D. C. G.; Fisk, A. T.; Cymbalisty, C. D.; Westmore, J. B. Quantifying $\mathrm{C}_{10}-\mathrm{C}_{13}$ polychloroalkanes in environmental samples by high-resolution gas chromatography electron capture negative ion high resolution mass spectrometry. Anal. Chem. 1997, 69 (14), 2762-2771.
(8) Zencak, Z.; Oehme, M. Recent developments in the analysis of chlorinated paraffins. TrAC, Trends Anal. Chem. 2006, 25 (4), 310317.

(9) Bayen, S.; Obbard, J. P.; Thomas, G. O. Chlorinated paraffins: a review of analysis and environmental occurrence. Environ. Int. 2006, 32 (7), 915-929.

(10) Feo, M. L.; Eljarrat, E.; Barceló, D.; Barceló, D. Occurrence, fate and analysis of polychlorinated $\mathrm{n}$-alkanes in the environment. TrAC, Trends Anal. Chem. 2009, 28 (6), 778-791.

(11) Coelhan, M. Determination of short chain polychlorinated paraffins in fish samples by short column GC/ECNI-MS. Anal. Chem. 1999, 71 (20), 4498-4505.

(12) Reth, M.; Zencak, Z.; Oehme, M. New quantification procedure for the analysis of chlorinated paraffins using electron capture negative ionization mass spectrometry. J. Chromatogr. A 2005, 1081 (2), 225231

(13) Gao, Y.; Zhang, H. J.; Su, F.; Tian, Y. Z.; Chen, J. P. Environmental occurrence and distribution of short chain chlorinated paraffins in sediments and soils from the Liaohe River Basin, P. R. China. Environ. Sci. Technol. 2012, 46 (7), 3771-3778.

(14) Reth, M.; Oehme, M. Limitations of low resolution mass spectrometry in the electron capture negative ionization mode for the analysis of short- and medium-chain chlorinated paraffins. Anal. Bioanal. Chem. 2004, 378 (7), 1741-1747.

(15) Van Mourik, L. M.; Leonards, P. E.; Gaus, C.; De Boer, J. Recent developments in capabilities for analysing chlorinated paraffins in environmental matrices: A review. Chemosphere 2015, 136, 259272

(16) Zeng, L. X.; Wang, T.; Han, W.; Yuan, B.; Liu, Q.; Wang, Y.; Jiang, G. Spatial and Vertical Distribution of short chain chlorinated paraffins in soils from wastewater irrigated farmlands. Environ. Sci. Technol. 2011, 45 (6), 2100-2106.

(17) Pellizzato, F.; Ricci, M.; Held, A.; Emons, H.; Böhmer, W.; Geiss, S.; Iozza, S.; Mais, S.; Petersen, M.; Lepom, P. Laboratory intercomparison study on the analysis of short-chain chlorinated paraffins in an extract of industrial soil. TrAC, Trends Anal. Chem. 2009, 28 (8), 1029-1035.

(18) van der Veen, I.; Crum, S.; De Boer, J. Interlaboratory Study on the Analysis of Chlorinated Paraffins in Environmental Matrices Phase II; Report W-14/18, version 1; Institute for Environmental Studies: Amsterdam, 2014.

(19) Zencak, Z.; Reth, M.; Oehme, M. Determination of total polychlorinated $\mathrm{n}$-alkane concentration in biota by electron ionization MS/MS. Anal. Chem. 2004, 76 (7), 1957-1962.

(20) Bogdal, C.; Alsberg, T.; Diefenbacher, P. S.; MacLeod, M.; Berger, U. Fast quantification of chlorinated paraffins in environmental samples by direct injection high-resolution mass spectrometry with pattern deconvolution. Anal. Chem. 2015, 87 (5), 2852-2860.

(21) Korytár, P.; Haglund, P.; De Boer, J.; Brinkman, U. Comprehensive two-dimensional gas chromatography for the analysis of organohalogenated micro-contaminants. TrAC, Trends Anal. Chem. 2006, 25 (4), 373-396.

(22) Rowland, S. J.; Scarlett, A. G.; Jones, D.; West, C. E.; Frank, R A. Diamonds in the rough: identification of individual naphthenic acids in oil sands process water. Environ. Sci. Technol. 2011, 45 (7), 31543159.

(23) Pena-Abaurrea, M.; Jobst, K. J.; Ruffolo, R.; Shen, L.; McCrindle, R; Helm, P. A.; Reiner, E. J. Identification of potential novel bioaccumulative and persistent chemicals in sediments from Ontario (Canada) using scripting approaches with GCxGC-TOF MS analysis. Environ. Sci. Technol. 2014, 48 (16), 9591-9599.

(24) Dimitriou-Christidis, P.; Bonvin, A.; Samanipour, S.; Hollender, J.; Rutler, R.; Westphale, J.; Gros, J.; Arey, J. S. GCXGC Quantification of Priority and Emerging Nonpolar Halogenated Micropollutants in All Types of Wastewater Matrices: Analysis Methodology, Chemical Occurrence, and Partitioning. Environ. Sci. Technol. 2015, 49 (13), $7914-7925$

(25) Korytár, P.; Parera, J.; Leonards, P. E. G.; Santos, F. J.; De Boer, J.; Brinkman, U. A. T. Characterization of polychlorinated n-alkanes 
using comprehensive two-dimensional gas chromatography-electroncapture negative ionisation time-of-flight mass spectrometry. $J$. Chromatogr. A 2005, 1086 (1-2), 71-82.

(26) Xia, D.; Gao, L. R.; Zhu, S.; Zheng, M. H. Separation and screening of short-chain chlorinated paraffins in environmental samples using comprehensive two-dimensional gas chromatography with micro electron capture detection. Anal. Bioanal. Chem. 2014, 406 (29), 7561-7570.

(27) Tranchida, P. Q.; Franchina, F. A.; Dugo, P.; Mondello, L. Comprehensive two-dimensional gas chromatography-mass spectrometry: Recent evolution and current trends. Mass Spectrom. Rev. 2014, DOI: $10.1002 /$ mas.21443.

(28) Ubukata, M.; Jobst, K. J.; Reiner, E. J.; Reichenbach, S. E.; Tao, Q.; Hang, J.; Wu, Z.; Dane, A. J.; Cody, R. B. Non-targeted analysis of electronics waste by comprehensive two-dimensional gas chromatography combined with high-resolution mass spectrometry: Using accurate mass information and mass defect analysis to explore the data. J. Chromatogr. A 2015, 1395, 152-159.

(29) Gao, Y.; Zhang, H. J.; Chen, J. P.; Zhang, Q.; Tian, Y. Z.; Qi, P. P.; Yu, Z. K. Optimized cleanup method for the determination of short chain polychlorinated $n$-alkanes in sediments by high resolution gas chromatography/electron capture negative ion-low resolution mass spectrometry. Anal. Chim. Acta 2011, 703 (2), 187-193.

(30) Zencak, Z.; Borgen, A.; Reth, M.; Oehme, M. Evaluation of four mass spectrometric methods for the gas chromatographic analysis of polychlorinated n-alkanes. J. Chromatogr. A 2005, 1067 (1-2), 295301.

(31) Tomy, G. T.; Stern, G. A. Analysis of $\mathrm{C}_{14}-\mathrm{C}_{17}$ polychloro-nalkanes in environmental matrixes by accelerated solvent extractionnigh-resolution gas chromatography/electron capture negative ion high-resolution mass spectrometry. Anal. Chem. 1999, 71 (21), 48604865.

(32) Gao, Y.; Zhang, H. J.; Su, F.; Tian, Y. Z.; Chen, J. P. Environmental occurrence and distribution of short chain chlorinated paraffins in sediments and soils from the Liaohe River Basin, P. R. China. Environ. Sci. Technol. 2012, 46 (7), 3771-3778.

(33) Chen, M. Y.; Luo, X. J.; Zhang, X. L.; He, M. J.; Chen, S. J.; Mai, B. X. Chlorinated paraffins in sediments from the Pearl River Delta, South China: spatial and temporal distributions and implication for processes. Environ. Sci. Technol. 2011, 45 (23), 9936-9943.

(34) Yuan, B.; Wang, T.; Zhu, N. L.; Zhang, K. G.; Zeng, L. X.; Fu, J. J.; Wang, Y. W.; Jiang, G. B. Short chain chlorinated paraffins in mollusks from coastal waters in the Chinese Bohai Sea. Environ. Sci. Technol. 2012, 46 (12), 6489-6496.

(35) Wang, T.; Han, S. L.; Yuan, B.; Zeng, L. X.; Li, Y. M.; Wang, Y. W.; Jiang, G. B. Summer-winter concentrations and gas-particle partitioning of short chain chlorinated paraffins in the atmosphere of an urban setting. Environ. Pollut. 2012, 171, 38-45.

(36) Zeng, L. X.; Zhao, Z. S.; Li, H. J.; Wang, T.; Liu, Q.; Xiao, K.; Du, Y. G.; Wang, Y. W.; Jiang, G. B. Distribution of short chain chlorinated paraffins in marine sediments of the East China Sea: influencing factors, transport and implications. Environ. Sci. Technol. 2012, 46 (18), 9898-9906. 